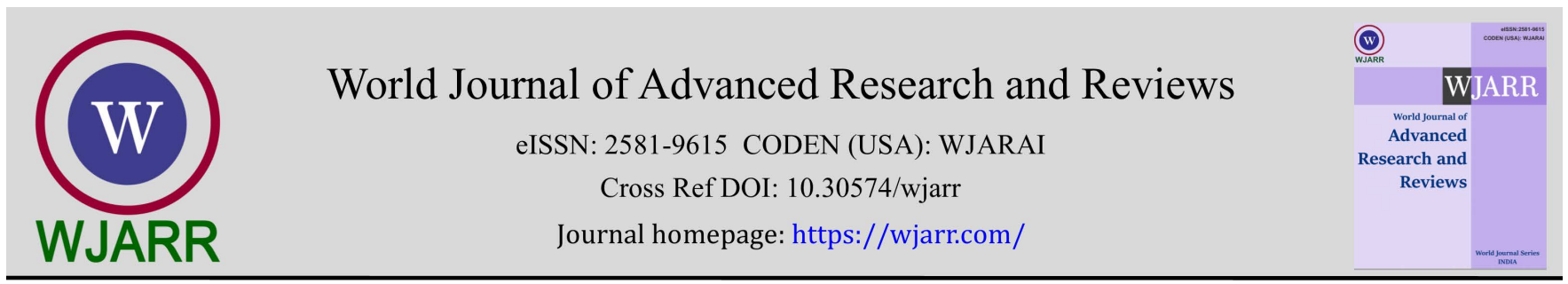

(RESEARCH ARTicle)

Check for updates

\title{
Biological synthesis and characterization of copper oxide nanoparticles using aqueous Psidium guajava leave extract and study of antibacterial activity of the copper oxide nanoparticles on Escherichia coli and Staphylococcus aureus
}

\author{
Samuel Paul 1,*, Akintunde Sheyi 2 , Iliya Daniel Bangu ${ }^{3}$, Sani Idris Abubakar ${ }^{3}$ and Muawiyya Muazu \\ Muhammad 4 \\ ${ }^{1}$ Department of Chemical Sciences, Federal University, Kashere P.M.B 0182 Gombe State Nigeria. \\ ${ }^{2}$ Department of Science Laboratory Technology, Federal College of Forestry Jos, P.M.B 2019, Plateau State, Nigeria. \\ ${ }^{3}$ Department of Soil Science, Federal University, Kashere P.M.B 0182 Gombe State Nigeria. \\ ${ }^{4}$ Department of Forestry Management and Research, Upland Forest Research Station Gombe, P.M.B 5054 Gombe State \\ Nigeria.
}

World Journal of Advanced Research and Reviews, 2021, 09(01), 114-120

Publication history: Received on 23 December 2020; revised on 02 January 2021; accepted on 03 January 2021

Article DOI: https://doi.org/10.30574/wjarr.2021.9.1.0513

\begin{abstract}
Nanobiotechnogy has been developed to synthesized copper oxide nanoparticle. This was carried out biologically using leaf extract of Psidium guajava as a reducing and stabilizing agent. Optical property showed a color change from dark brown to gold- yellow after the addition of leaf extract. The possible functional group in the plant extract responsible for the reduction of CuONps was identified as belonging to alkyl halide group by FT-IR analysis. UV- visible spectra of copper oxide nanoparticle showed a surface plasmon resonance peak at 390 $\mathrm{nm}$. The biologically synthesized copper nanoparticles were tested for antibacterial activity against two human pathogens viz, Escherichia coli and Staphylococcus aureus. Biosynthesized copper nanoparticle showed clear zone of inhibition or better activity against Escherichia coli and Staphylococcus aureus.
\end{abstract}

Keywords: Psidium guajava; Nanoparticles; Synthesis; UV-VIS; FT-IR

\section{Introduction}

Nano-chemistry is a branch of nanoscience which deals with the chemical application of nanomaterial in nanotechnology. It also involves the study of the synthesis and characterization of material on a Nano scale science [1].

Metal nanoparticles are currently an area of extreme scientific research due to a wide variety of potential application in biomedical, optical and electronic field. Metal nanoparticles are also of great interest due to their novel physicochemical, magnetic and optoelectronic properties which are governed by their size distribution [2]. It is predominantly due to it extremely small size and large surface area to volume ratio that lead to the significant differences in properties such as biological catalytic activity, mechanical melting point, optical absorption, thermal and electrical conductivity which are not seen in their bulk form [3]. Because of these unique physicochemical and optoelectronic properties, nanoparticles are of particular interest for a number of applications ranging from catalyst, chemical sensors, electronic components, medical diagnostic imaging, pharmaceuticals products and medical treatment protocols.

Green synthesis provides advancement over chemical and physical method as it is cost effective, environment friendly, easily scaled up for large-scale synthesis and further there is no need to use high pressure, energy, temperature and

\footnotetext{
* Corresponding author: Samuel Paul

Department of Chemical Sciences, Federal University, Kashere P.M.B 0182 Gombe State Nigeria.

Copyright $(2021$ Author(s) retain the copyright of this article. This article is published under the terms of the Creative Commons Attribution Liscense 4.0.
} 
toxic chemicals. Synthesis of NPs using biological entities has great interest due to their unusual optical, chemical, photo electrochemical and electronic properties [4].

In practice, the physical, chemical and biological approaches are employed for the synthesis of nanomaterial. Particularly, the biological methods like plant and microbial mediated process for the synthesis of nanoparticles are showing significant interest because of their less environmental consequences and energy intensive process. In biological methods using microorganisms, the titanium nanoparticles have been synthesized by either intra or extracellularly by bacteria yeast [5], and fungi [6]. The microbial mediated synthesis of nanoparticles requires maintenance of organisms and optimum parameters such as temperature, $\mathrm{pH}$ and other factors for microbial growth. In order to develop the simple green synthesis, the plant extract mediated process was considered as a suitable method for the synthesis of copper oxide nanoparticles.

Recently much work has been done with regard to plant assisted reduction of metal nanoparticle and the respective role of phytochemical. The advantage of using plants for the synthesis of nanoparticles is that they are easily available, safe to handle and possess a broad variability of metabolites that may aid in reduction. A number of plants are being currently investigated for their role in the synthesis of nanoparticles [7]. The main water soluble phytochemical are flavones, organic acids and quinines which are responsible for immediate reduction [8].

\section{Material and methods}

Guava (Psidium guajava) leaves, copper sulphate anhydrous, Hannah instrument 2211 pH meter, Jenway 6400 UVVisible spectrophotometer, Perkin Elmer Frontier Fourier Transform Infrared Infrared spectroscopy and ultrasonic bath.

\subsection{Preparation of the leaf extract}

Psidium guajava plant was locally collected at Adamawa State University $9.3265^{\circ} \mathrm{N}, 12.3984^{0} \mathrm{E} .10 \mathrm{~g}$ was washed with distilled water to remove dust and cut into pieces, placed in a $200 \mathrm{~mL}$ Erlenmeyer flask and boiled with distilled water for 20 minutes, and allow to cool under room temperature. The solution was then filtered with Whatman filter paper No. 1 to obtain extract.

\subsection{Pharmocognostic evaluation of aqueous extract}

Fresh extract of the fruit of Psidium guajava was used for phytochemical Screening-Qualitative analysis.

\subsubsection{Phytochemical Screening-Qualitative Analysis}

Preliminary phytochemical Screening was conducted for the identification of carbohydrates, alkaloids, tannins, steroids, flavonoids, glycosides, saponins, triterpenoids and phenol using standard phytochemical method [9]

\subsection{Preparation of copper nanoparticles}

$0.042 \mathrm{~g}$ of copper sulphate anhydrous was weighed with a top load balance and was dissolved in $100 \mathrm{~mL}$ distilled water to prepare a $0.001 \mathrm{M}$ copper sulphate anhydrous solution. $60 \mathrm{~mL}$ of copper sulphate anhydrous was added to $5 \mathrm{~mL}$ of leaf extract and stirred using electric stirrer for 10 minutes at room temperature. Change in color of the solution indicates the formation of copper oxide nanoparticles. The product formed was centrifuged at $3000 \mathrm{rpm}$ for $10 \mathrm{minutes}$, washed with ethanol and distilled water and was allowed to dry under room temperature. [10].

\subsection{Determination of antibacterial activity}

Antibacterial activity of the extract was determined on Muller and Hinton Agar using Kirby-Bauer disk diffusion method [11]. Test pathogens were spread on the test plates- Muller Hinton Agar (MHA) for bacterial using sterile swabs. Sterile

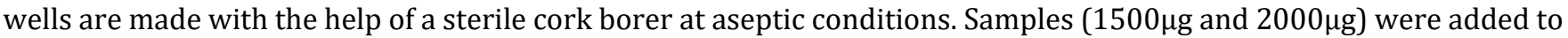
the wells at aseptic conditions. Stock solutions of the extract were prepared using DMSO. The test plates were incubated for $24 \mathrm{hrs}$. The zone of inhibition (in mm diameter) were read and taken as the activity of the extract against the test organisms. 


\section{Results and discussion}

Table 1 qualitative phytochemical screening of fresh psidium quajava

\begin{tabular}{|l|l|l|}
\hline Sr. No. & Phytochemical & Results \\
\hline 1 & Carbohydrate & + \\
\hline 2 & Flavonoids & + \\
\hline 3 & Alkaloids & + \\
\hline 4 & Steroids & + \\
\hline 5 & Glycosides & + \\
\hline 6 & Tannins & - \\
\hline 7 & Saponins & - \\
\hline 8 & Triterpenoids & + \\
\hline 9 & Phenols & + \\
\hline \multicolumn{2}{|l}{ Indication of sigh (+) present (-) absent } \\
\hline
\end{tabular}

Table 2 FT-IR Analysis of Psidium guajava leaf

\begin{tabular}{|l|l|l|l|l|}
\hline S/N & Class of Compound & Absorption $\left(\mathrm{cm}^{-1}\right)$ & Intensity & Assignment \\
\hline 1. & Alcohol, O-H & 3225.32 & Strong & O-H (Stretching, H bonding \\
\hline 2. & Alkane, C-H & 2914.85 & Strong & C-H (Stretching) \\
\hline 3. & Alkyne, $-\mathrm{C}=\mathrm{C}-$ & 2115.05 & Variable & -C=C- (Stretching) \\
\hline 4. & Amine, C-N & 1198.84 & Medium, Weak & C-N (Stretching) \\
\hline 5. & Aromatic alkene, C=C & 1604.29 & Medium-Broad & C=C (Stretching) \\
\hline 6. & Aromatic, C=C & 1443.01 & Medium, Weak & C=C (Stretching) \\
\hline 7. & Alkyl halide, C-F & 1036.30 & Strong & C-F (Stretching) \\
\hline 8. & Amide, N-H & 1515.71 & Strong & N-H (Bending) \\
\hline 9. & Alkene $=$ C-H & 764.22 & Strong & C-H (Bending) \\
\hline
\end{tabular}

Table 3 FT-IR analysis of copper oxide nanoparticle synthesized from Psidium guajava leaf

\begin{tabular}{|l|l|l|l|l|}
\hline S/N & Class of Compounds & Absorption $\left(\mathrm{cm}^{-1}\right)$ & Intensity & Assignment \\
\hline 1. & Alcohol, O-H & 3203.01 & Strong & Broad \\
\hline 2. & Acid, O-H & 2915.25 & Variable & Very broad \\
\hline 3. & Aldehyde, =C-H & 2847.82 & Medium & =C-H (stretching) \\
\hline 4. & Nitrile, CN & 2325.04 & Medium & CN (Stretching) \\
\hline 5. & N=C, Isonitriles & 2115.44 & Strong & N=C (Stretching) \\
\hline 6. & Substituted benzene ring & 1992.93 & Medium & $\begin{array}{l}\text { Several bands from } \\
\text { ketone }\end{array}$ \\
\hline 7. & Amine, N-H & 1600.38 & Medium & N-H (Stretching) \\
\hline 8. & Ester, C-O & 1036.74 & Strong & C-O (Stretching) \\
\hline
\end{tabular}


Table 4 Showing zone of inhibition against bacterial pathogens

\begin{tabular}{|l|l|l|l|}
\hline Culture name & Copper nanoparticle $1500 \mu \mathrm{g}$ & $2000 \mu \mathrm{g}$ & PC \\
\hline E. coli & 10.01 & 14.02 & 24.00 \\
\hline Staphylococcus aureus & - & - & 22.02 \\
\hline
\end{tabular}
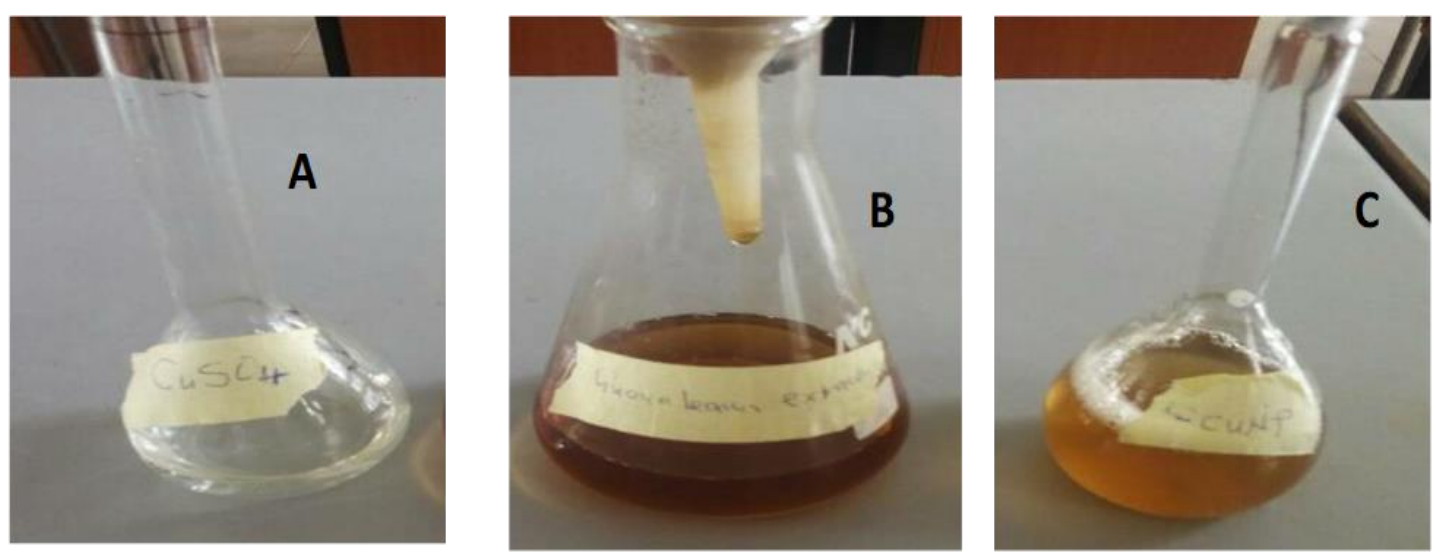

Figure 1 Optical behavior of copper sulphate anhydrous A, Psidium guajava leave extract B and copper oxide nanoparticle $\mathrm{C}$

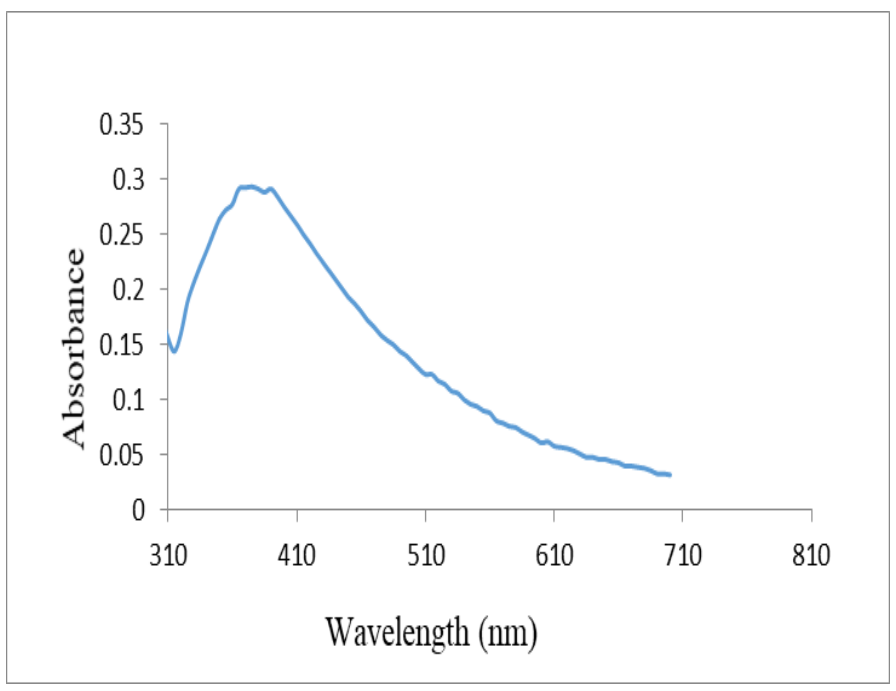

Figure 2 UV-visible spectrum of copper oxide nanoparticle synthesized using Psidium guajava. 


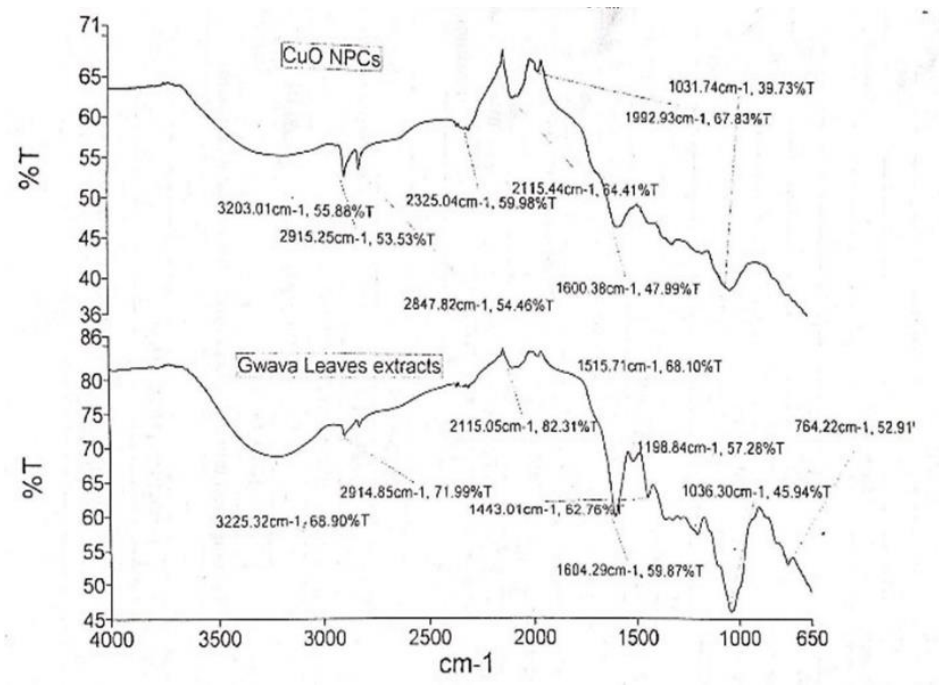

Figure 3 FT-IR absorption spectra of copper oxide nanoparticle synthesized from Psidium guajava leaf extract
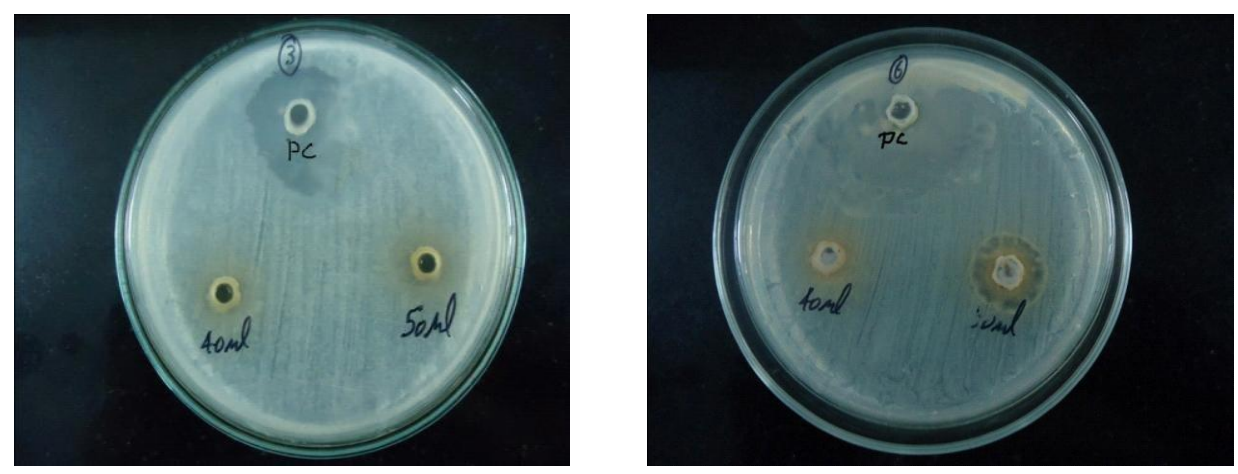

Figure 4 Antibacterial activities of CuNps or Zone of inhibition of green synthesis of CuNps against (a) E-Coli and (b) Staphylococcus aureus

\section{Discussion}

The results of quantitative phytochemical analysis of the Psidium guajava extract are shown in table 1 which indicates the process of secondary metabolites such as carbohydrate, flavonoids, alkaloids, steroids, glycosides, tannins, saponins, phenols and triterpenoids. The presence of ascorbic acid, polyphenols and other phytonutrients present in aqueous Psidium guajava extract is mainly responsible for the bio-reduction process [12]. From the literature it has been found that the amount of ascorbic acid (natural vitamin C) present in Psidium guajava extract was found to be $479 \mathrm{mg}$ of ascorbic acid/100gm of fruit-polyphenolic compounds are very important plant constituents because of the scavenging ability of their $-\mathrm{OH}$ groups. The antioxidant property of polyphenolic compound is mainly due to the redox property which allows them to act as reducing agents [13]

The change in colour in Fig. 1 of $0.001 \mathrm{M}$ copper sulphate anhydrous from colorless to dark brown after the addition of $5 \mathrm{~mL}$ of Psidium guajava leave extract and then to gold-yellow colour indicates the formation of copper oxide nanoparticle. Therefore, copper oxide nanoparticle is known to exhibit characteristic gold-yellow colour.

Fig. 2 shows the UV-visible spectrum information on copper oxide nanoparticle by reduction with metal ion when exposed to Psidium guajava extract. The UV-visible absorption spectrum of copper oxide nanoparticle was scanned from $300 \mathrm{~nm}$ to $700 \mathrm{~nm}$; it shows the characteristics surface Plasmon resonance (SPR) with absorbance at approximately $390 \mathrm{~nm}$ which can be ascribed to the formation of copper oxide nanoparticle.

The Fourier transform infrared spectroscopy analysis was carried out to identify the various possible biomolecules responsible for the bio reduction of copper ion and capping of the reduced copper oxides synthesized using Psidium 
guajava. Fig. 3 shows the spectra's of Psidium guajava and copper oxide nanoparticles. The result observed from Psidium guajava leave gave the IR band are $3225.32 \mathrm{~cm}^{-1}, 2914.85 \mathrm{~cm}^{-1}, 1443.01 \mathrm{~cm}^{-1}, 1604.04 \mathrm{~cm}^{-1}, 1998.84 \mathrm{~cm}^{-1}, 1036.30 \mathrm{~cm}^{-1}$, $2115.05 \mathrm{~cm}^{-1}$ and $764.22 \mathrm{~cm}^{-1}$. The band which appeared at $3225.32 \mathrm{~cm}^{-1}$ corresponds to $0-\mathrm{H}$ stretching, H-bonded. The bands at $2914.85 \mathrm{~cm}^{-1}$ are due to $\mathrm{C}-\mathrm{H}$ stretching and symmetric stretch. The band at $2115 \mathrm{~cm}^{-1}$ are due to $-\mathrm{C}=\mathrm{C}-$ stretching. The band at $1198.84 \mathrm{~cm}^{-1}$ are due to the IR band at $1604.29 \mathrm{~cm}^{-1}$ gave $\mathrm{C}=\mathrm{C}$ stretching, IR band observed at $1443.01 \mathrm{~cm}^{-1}$ gave $\mathrm{C}=\mathrm{C}$ stretching. IR band at $1036.30 \mathrm{~cm}^{-1}$ gave $\mathrm{C}-0$ stretching, the IR band at $764.22 \mathrm{~cm}^{-1}$ gave $\mathrm{C}-\mathrm{Cl}$ stretching. The band at $1515.71 \mathrm{~cm}^{-1}$ which is absent in the spectra of synthesized copper oxide nanoparticle which was assigned to the $\mathrm{N}-\mathrm{H}$ bending stretching amide group may be responsible for the reduction CuONps. And the band at $764.22 \mathrm{~cm}^{-1}$ assigned to $\mathrm{C}-\mathrm{Cl}$ stretching belonging to Alkyl halide group is also responsible for the reduction of CuONps.

Antimicrobial activity of biosynthesized copper nanoparticles was examined out on two pathogens, such as E. coli (gram -ve) and Staphylococcus aureus (gram +ve). Biosynthesized copper nanoparticle showed clear zone of inhibition as indicated in the table 4 against E. coli and Staphylococcus aureus. It is reported that Copper nanoparticles attach to the surface of the cell membrane, disturbs its function and penetrates directly with the bacterial outer membrane and release copper ions. Ciprofloxacin $25 \mu \mathrm{g} / \mathrm{ml}$ was used as +ve control

\section{Conclusion}

The biosynthesis of copper oxide nanoparticle from copper sulphate anhydrous using Psidium guajava leave extract as a reducing agent was successfully carried out. The presence of copper oxide nanoparticle was studied and confirmed using various techniques such as optical behavior, and spectroscopy. UV-visible spectroscopy gave an observed wavelength of copper oxide nanoparticle at $380 \mathrm{~nm}$. FT-IR spectroscopy showed the phytochemical responsible for the reduction of $\mathrm{CuONps}$ which belong to alkyl halide group. The copper nanoparticle showed clear zone of inhibition against E. coli and Staphylococcus aureus. This study conclusively reports an eco-friendly approach for synthesis of copper oxide nanoparticles. Such studies have potential for developing good antibacterial formulations having nanoparticles.

\section{Compliance with ethical standards}

\section{Acknowledgments}

This research did not receive any form of grant from government or non-governmental organization

\section{Disclosure of conflict of interest}

The authors declare that they have no known competing financial interests or personal relationships that could have appeared to influence the work reported in this paper

\section{References}

[1] Doc Brown's.chemistry Revision Notebook dopamine. Journal of sensors and Actuators B. 2011; 93-102.

[2] Daniel MC, Astruc D. Gold nanoparticle; Assembly, supramolecular chemistry,quantum-size-related properties, and applications toward biology, catalysis, and nanotechnology. J. Chem. Rev. 2004; 104: 293-396.

[3] Perez J, Bax L, Escolano C. Roadmap report on Nanoparticles; Willemsy and Van Den Wilddenberg: Barcelona, spain. 2005.

[4] Mohanpuria, NK, Rama, SK Yadav. Biosynthesis of nanoparticles: technology concepts for feature Application. J, NPS. 2008; 10: 507-517.

[5] Jhaa A, Prasad K. A green low-cost biosynthesis of $\mathrm{Sb}_{2} \mathrm{O}_{3}$ nanoparticles. 2009; 43(3): 303-306

[6] Rajakumar G, Abdul RA, Mohana RS, Gopiesh KV, Elango G, Kamaraj C, Abduz ZA, Velayutham K. Fungus-mediated biosynthesis and characterization of $\mathrm{TiO} 2$ nanoparticles and their activity against pathogenic bacteria. SpectrochemActa A. 2012; 91: 23-29.

[7] Pranthna T, Lazar M, Chandrasekaran A, Raichur, Amitava M. Biomimetic synthesis of nanoparticles Science Technology \& Applicability. Biomimetics learning from nature. 2010; 953-978.

[8] Sushmita C, Latika S, Manoranjan PS. Phytochemical and antibacterial screening of Psidium guajava L. leaf extracts against clinically important gastrointestinal pathogens. Ranchi. 2012; 2(4): 524-529. 
[9] Caroling G, Vinodhini E, Mercy, Ranjitham A, Shanthi P. Biosynthesis of copper nanoparticles using aqueous phyllanthus embilica (gooseberry) extract-characterization and study of antimicrobial effects. Int. J. Nano Chem. 2015; 1(2): 53-63.

[10] Baur AW, Kirby VMM, Sherris JC, Turck M. Antibiotic susceptibility testing by a standardized single disk method. Am J clinpathol. 1996; 45: 493-496.

[11] Bartner A, Pfeiffern KP, Bartner H. Applicability of disc diffusion methods required by the pharmacopoeias for testing antibacterial activity of natural compounds. Pharmazie. 1994; 49: 512-516. 